\title{
Spectroscopic and Ultrasonic Exploration on Molecular Interaction of Ciprofloxacin with L-Glutamic Acid in Aqueous Medium
}

ISSN: 2576-8840

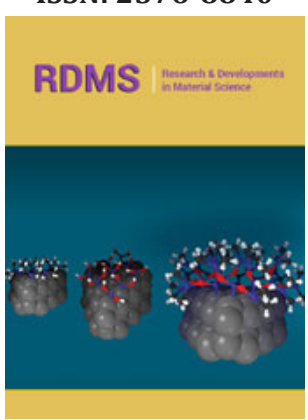

*Corresponding author: Patnaik $\mathrm{AK}$ Department of Chemistry, Ravenshaw University, Cuttack, Odisha-753003, India

Submission: 梅 September 28, 2019

Published: 侮 October 11, 2019

Volume 12 - Issue 1

How to cite this article: Sahoo A, Das S, Patnaik A. Spectroscopic and Ultrasonic Exploration on Molecular Interaction of Ciprofloxacin with L-Glutamic Acid in Aqueous Medium. Res Dev Material Sci. 12(1).RDMS.000776.2019.

DOI: $10.31031 /$ RDMS.2019.12.000776

Copyright@Patnaik AK, This article is distributed under the terms of the Creative Commons Attribution 4.0 International License, which permits unrestricted use and redistribution provided that the original author and source are credited.

\author{
Sahoo $\mathrm{AK}^{1}$, Das $\mathrm{SP}^{2 *}$ and Patnaik $\mathrm{AK}^{2 *}$ \\ ${ }^{1}$ Department of Chemistry, Christ College, Cuttack, India \\ ${ }^{2}$ Department of Chemistry, Ravenshaw University, India
}

\begin{abstract}
Studies on molecular interactions using ultrasonic and spectroscopic technique in ternary liquid mixture of drug ciprofloxacin, a proteinogenic amino acid l-glutamic acid in aqueous medium has been carried out at $300.15 \mathrm{~K}$ and $2 \mathrm{MHz}$ frequency. Different acoustical parameters such as adiabatic compressibility ( $\beta$ ), intermolecular free length (Lf), acoustic impedance (z) etc are evaluated using experimental values of density $(\rho)$ and ultrasonic velocity $(U)$ These parameters along with UV-Visible spectroscopic evidences demonstrates drug-amino acid molecular interaction existing in the aqueous media.
\end{abstract}

Keywords: Ciprofloxacin; L-glutamic acid; Adiabatic compressibility; Intermolecular free length; Acoustic impedance

Introduction

Ciprofloxacin is a second-generation fluoroquinolone with a broad spectrum of activity and is used to treat a wide variety of infections, including infections of bones and joints, endocarditis, gastroenteritis, malignant otitis externa, respiratory tract infections, cellulitis, urinary tract infections, prostatitis, anthrax, and chancroid [1]. It is on the World Health Organization's List of Essential Medicines, the most effective and safe medicines needed in a health system. This compound was found to be substantially more active against enterobacteriaceae than the older drugs of this class, such as nalidixic acid, with minimum inhibitory concentrations ranging from 0.008 to $2.0 \mathrm{mg} / \mathrm{L}$. A single-dose pharmacokinetic study of ciprofloxacin showed that an oral dose of ciprofloxacin was absorbed well and rapidly and exhibited excellent tissue penetration $[2,3]$.

In addition to biosynthesis of proteins, glutamic acid has several key functions within the body. This amino acid (also called 'chemical messenger') is an excitatory neurotransmitter increasing the firing of neurons in the human central nervous system $[4,5]$. Being one of the few nutrients able to pass through the blood-brain barrier, this amino acid appears to support brain function. In other words, Glutamic acid turned out to be human brain's primary 'food'. When it reaches the brain, it utilizes all excess ammonia, which is a toxic waste product of metabolism, by transforming it into the amino acid called Glutamine. This amino acid has been used to help treat Parkinson's, fatigue, mental retardation, schizophrenia, muscular dystrophy, and alcoholism. Besides, it is believed to help shuttle potassium (an important mineral) across the blood-brain barrier and right into the spinal fluid. Besides, Glutamic acid is acting as an intermediary in the Kreb's cycle and is important for the carbohydrate metabolism as well as detoxifying muscle cells [6-8].

A lot of interest has been evoked by the recognition of drugs activities in aqueous and nonaqueous solutions, which involve the interactions with biological membranes. Physiologically, the consequences of a drug's action such as movement of the drug through the bloodstream, distributions, receptor binding, and lastly, desired actions involve an intricate mechanism which can be perceived by knowing the thermodynamic behaviour of such systems indicated by different molecular interactions $[9,10]$. Ultrasonic is a versatile nondestructive technique and is highly useful to interpret drug-amino acid macromolecular interaction which plays an important role in the understanding the mode of drug action through intra and extracellular fluids. 


\section{Experimental}

\section{Materials}

The drug ciprofloxacin was procured from Sigma Aldrich (mass fraction purity 0.987). l-glutamic acid (mass fraction $>0.99$ ) was obtained from Himedia Ltd. Doubly distilled deionised water was used in our experiments.

\section{Methods}

The densities of the solutions were measured using a single stem bicapillary Pycnometer (Borosil glass). An analytical balance (SHIMADZU AX- 200, Japan) with a precision of $\pm 0.1 \mathrm{mg}$ was used for all weight measurements. The ultrasonic speeds in solutions were measured using a single crystal variable path multifrequency ultrasonic interferometer [M-81S, Mittal Enterprises, India] operated at a fixed frequency of $2 \mathrm{MHz}$. All measurements were carried out in an automatic digital temperature controlled high precision water thermostat maintained at the temperature of $300.15 \mathrm{~K}$. UV-Visible spectra of the pure components and the ternary mixture was recorded through Agilent Cary 100 UV-Visible spectrophotometer.

In our present work different acoustical and volumetric properties such as density, ultrasonic velocity have been measured for ciprofloxacin hydrochloride drug with l-glutamic acid in aqueous medium at $300.15 \mathrm{~K}$ and different ultrasonic parameters such as adiabatic compressibility $(\beta)$, intermolecular free length $\left(L_{f}\right)$, acoustic impedance(z) etc. have been discussed to interpret solutesolute and solvent-solvent interactions and also to have insight about the structure making and structure-breaking capability of drug and the biomolecule [11].

\section{Result and Discussion}

Figure 1 shows comparative spectra of ciprofloxacin, l-glutamic acid in aqueous medium. ciprofloxacin showed two peaks at $280 \mathrm{~nm}$. and $320 \mathrm{~nm}$ while l-glutamic acid showed a single peak at $320 \mathrm{~nm}$. But the UV spectra of binary mixture shows the complete disappearance of the peak of glutamic acid which indicates association of ciprofloxacin with glutamic acid in the mixture.

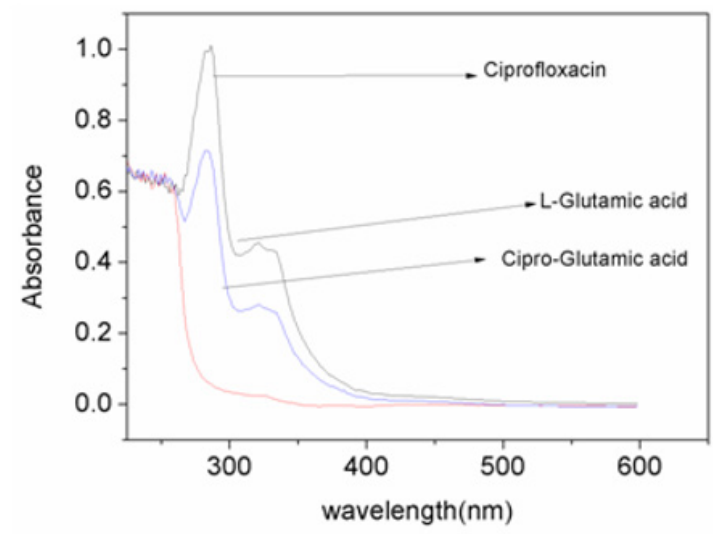

Figure 1: Comparative study of spectra of ciprofloxacin with L-glutamic acid.
From the analysis of variation of density of solution as a function of concentration (Figure 2) of ciprofloxacin and L-glutamic acid, it is observed that density of solution increases gradually with concentration but there is a sharp increase of density in case of ciprofloxacin than L-glutamic acid $[12,13]$.

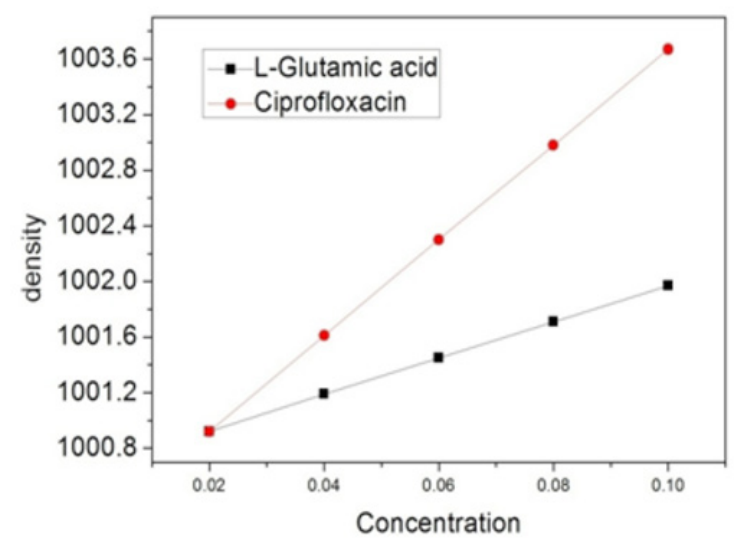

Figure 2: Variation of density with concentration.

Variation in ultrasonic velocity with concentration (Figure 3) indicates the presence of intermolecular interactions between the drug-amino acid mixture and solvent molecules [14]. It is noticed that value of $U$ increases with the increase in concentration of solution. The increasing concentration of drugs supports non rupturing of drug molecules and hence there is an increase in ultrasonic velocity in bulk of solution. We can see steady increase in case of L-glutamic acid, but ciprofloxacin shows sharp increase than L-glutamic acid at concentration of $0.08 \mathrm{M}$.

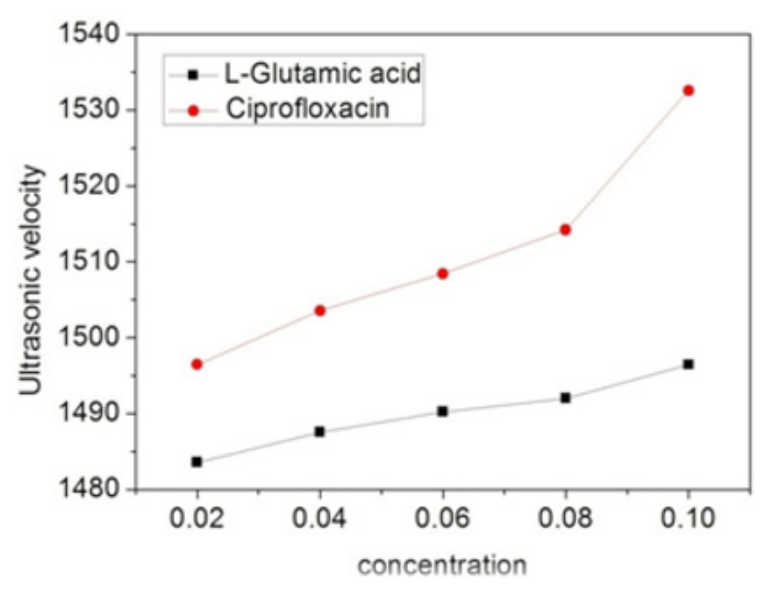

Figure 3: Variation of ultrasonic velocity with concentration.

It is seen from Figure 4 that the value of adiabatic compressibility decreases with the increase of solute concentration for both the systems and can be attributed to the fact that with increase in concentration the decrease of $\beta$ value might be due to aggregation of solvent molecules around solute particles indicating thereby the presence of solute- solvent interactions in all these systems. Smooth decrease is seen in both except for cipro at $0.08 \mathrm{M}$. 


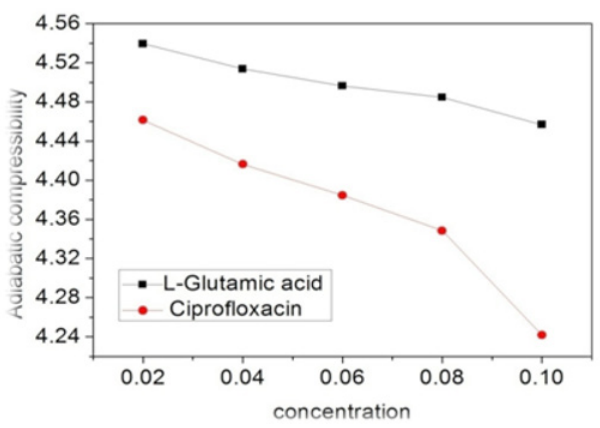

Figure 4: Variation of adiabatic compressibility with concentration.

Figure 5 represents the plots of intermolecular free length (Lf) of the solution versus concentration of the solution. It is evident that the value of the ultrasonic velocity depends on intermolecular free length. The velocity increases with decrease in Lf or vice versa. Lf decreases continuously which suggest that there is strong interaction between solvent and drug molecules.

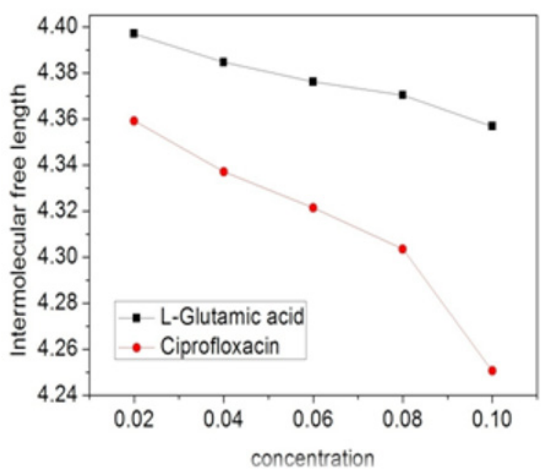

Figure 5: Variation of intermolecular free length with concentration.

Figure 6 depicts the variation of acoustic impedance $(\mathrm{Z})$ of solution as a function of molarity (c) of solution. The increase of $\mathrm{Z}$ with solute concentration can be attributed to the effective solventsolute interactions and weaker solute-solute interaction. Cipro shows more increase which is sharper at $0.08 \mathrm{M}$.

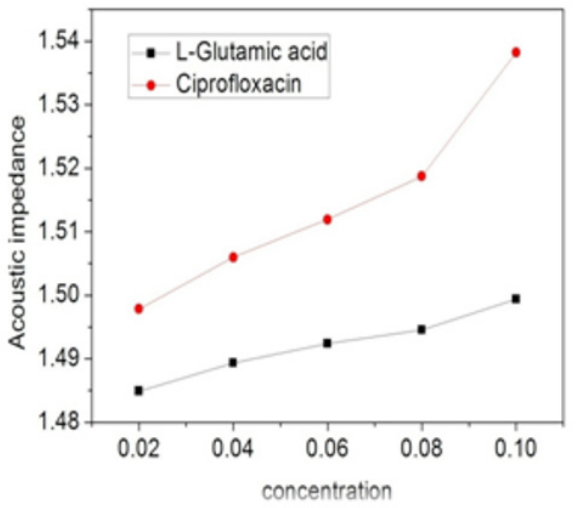

Figure 6: Variation of acoustic impedance with concentration.

\section{Conclusion}

A detailed analysis of various interaction parameters such as acoustic impedance, intermolecular free length, adiabatic compressibility etc for the binary system of antibiotic drug ciprofloxacin and non-essential amino acid L-Glutamic acid in aqueous medium, it can be concluded that L-Glutamic acid shows associative solvation with the polar protic solvent water for all concentration range of amino acid. But in case of the drug ciprofloxacin the ultrasonic parameters furnish a linear up or down trend up to $0.08 \mathrm{M}$ concentration indicating associative interaction with glutamic acid and beyond 0.08M there is a sharp change in the trend. This suggests that at higher concentration association between drug -solvent, amino acid-solvent and drug-amino acid is more prominent and significant attachment with biological target can be easily possible. UV- VIS spectral studies show the disappearance of peak of glutamic acid and slight shifting of peak of ciprofloxacin with lower absorbance which indicates strong association between the drug with glutamic acid in aqueous medium. Clinically it is observed that by intake of ciprofloxacin, the side effects such as swelling, tenderness, stiffness, or loss of movement in joints, severe dizziness appear due to the deficiency of L-Glutamic acid caused by the antibiotic. Ultrasonic and spectral experimental data shows stronger association of ciprofloxacin with glutamic acid. Therefore, pharmacologically L-Glutamic acid should be prescribed as a supplement with ciprofloxacin in order to compensate the deficiency of glutamic acid to overcome the side effects of the intake antibiotic.

\section{References}

1. Barry AL, Jones RN, Thornsberry C, Ayers LW, Gerlach EH, et al. (1984) Antibacterial activities of ciprofloxacin, norfloxacin, oxolinic acid, cinoxacin and nalidixic acid. Antimicrob Agents Chemother 25: 633-637.

2. Darkopoulos AI, Ioannou PC (1997) Spectrofluorimetric study of the acid-base equilibria and complexation behavior of the fluoroquinolone antibiotics ofloxacin, norfloxacin, ciprofloxacin and pefloxacin in aqueous solution. Anal Chim Acta 354(1-3): 197-204.

3. Davis R, Markham A, Balfour JA (1996) Ciprofloxacin. An updated review of its pharmacology, therapeutic efficacy and tolerability. Drugs 51(6): 1019-1074.

4. Kumar H, Behal I (2016) Densities and speeds of sound of antibiotic drug chloramphenicol with l-Leucine and glycyl-l-leucine in aqueous medium at $\mathrm{T}=(288.15-318.15) \mathrm{K}:$ A Volumetric, Ultrasonic, and UV Absorption Study. J Chem Eng Data 61: 3740-3751.

5. Rodante F, Marrosu G (1989) Thermodynamics of the second proton dissociation processes of nine $\alpha$-amino-acids and the third ionization processes of glutamic acid, aspartic acid and tyrosine. Thermochimica Acta 141: 297-303.

6. Lehmann, Mogens S, Koetzle, Thomas F, Hamilton, Walter C (1972) Precision neutron diffraction structure determination of protein and nucleic acid components. VIII: the crystal and molecular structure of the $\beta$-form of the amino acid l-glutamic acid. Journal of Crystal and Molecular Structure 2(5): 225-233.

7. Meldrum BS (2000) Glutamate as a neurotransmitter in the brain: Review of physiology and pathology. The Journal of Nutrition. 130 (4S Suppl): 1007S-1015S.

8. Okubo Y, Sekiya H, Namiki S, Sakamoto H, Iinuma S, et al. (2010) Imaging extrasynaptic glutamate dynamics in the brain. Proceedings of the National Academy of Sciences 107(14): 6526-6531. 
9. Iqbal MJ, Chaudhry MA (2010) Effect of temperature on volumetric and viscometric properties of some non-steroidal antiinflammatory drugs in aprotic solvents. J Chem Thermodyn 42(8): 951-956.

10. Taboada P, Attwood D, Ruso JM, Garcia M, Mosquera V (2001) Thermodynamic properties of some antidepressant drugs in aqueous solution. Langmuir 17(1): 173-177.

11. Terdale SS, Dagade DH, Patil KJ (2007) Thermodynamic studies of drug-a-cyclodextrin interactions in water at $298.15 \mathrm{~K}$ : promazine hydrochloride/chlorpromazine hydrochloride? a-cyclodextrin? $\mathrm{H}_{2} \mathrm{O}$ systems. J Phys Chem B 111: 13645-13652.
12. Rajagopal K, Jayabalakrishnan SS (2010) Volumetric, ultrasonic speed, and viscometric studies of salbutamol sulphate in aqueous methanol solution at different temperatures. J Chem Thermodynamics 42(8): 984993.

13. Naik RR, Bawankar SV, Tekade PV, Mahodaya Om A (2015) Ultrasonic study of pyridoxine solutions at different temperatures and concentrations. Russian Journal of Physical Chemistry 89(1): 152-158.

14. Nalle PB, Birajdar SD, Shinde BR, Dorik RG, Jadhav KM (2016) Effect of drug Piper nigrum on physicochemical properties of zinc chloride at varying concentration and temperature investigated through ultrasonic tool. Cogent Chemistry 2(1): 1216721. 